\title{
Efeitos do Treinamento Muscular Inspiratório na Biomecânica da Deglutição de Pacientes com Bronquiectasia: Série de Casos
}

\author{
Bruna Franciele da Trindade Gonçalves, ${ }^{1}$ Marisa Bastos Pereira, ${ }^{2}$ Renata Mancopes ${ }^{2}$
}

\begin{abstract}
RESUMO
Objetivo: Analisar os efeitos do Treinamento Muscular Inspiratório na deglutição de sujeitos com bronquiectasia. Método: Ensaio clínico experimental randomizado não cegado. Os participantes realizaram avaliação clínica da deglutição, videofluoroscopia e manovacuometria. A amostra foi dividida em dois grupos: o grupo controle realizou fisioterapia respiratória convencional e o grupo estudo associou essa ao treinamento muscular inspiratório com POWERbreathe. Resultados: Foram avaliados 11 indivíduos com bronquiectasia, a maioria com deglutição normal (63,6\%), adultos de meia idade (54,5\%), do sexo feminino (72,7\%). Houve boa concordância entre os juízes que analisaram a biomecânica da deglutição antes e após as intervenções. Em ambos os grupos a PImáx foi maior após intervenção ( $\mathrm{P}=0,007)$. A PImáx, entretanto, foi superior no grupo estudo em relação ao grupo controle $(P=0,034)$. Houve melhora em ambos os grupos em algumas variáveis, como atraso do início da fase faríngea e resíduos em valéculas, embora sem significância estatística. Conclusão: A realização do treinamento muscular inspiratório não gerou efeitos significativos sobre a biomecânica da deglutição.
\end{abstract}

Palavras-chave: Bronquiectasia. Deglutição. Transtornos da deglutição. Reabilitação.

\section{EFFECTS OF INSPIRATORY MUSCLE TRAINING IN THE BIOMECHANICS OF DEGLUTITION} IN PATIENTS WITH BRONCHIECTASIA: SERIE OF CASES

\begin{abstract}
Objective: To analyze the effects of Inspiratory Muscular Training on the swallowing of subjects with bronchiectasis. Method: Unblinded randomized experimental clinical trial. Participants performed clinical evaluation of swallowing, videofluoroscopy and manovacuometry. The sample was divided into two groups, the control group who realized conventional respiratory physiotherapy and a study group that associated this whit the inspiratory muscle training with POWERbreathe. Results: Elavuated 11 individuals with bronchiectasis, most of them with normal swallowing (63.6\%), middle-aged adults (54.5\%) female (72.7\%). There was good agreement among the judges who analyzed the biomechanics of swallowing before and after the interventions. In both groups the IPmax was higher after intervention $(P=0.007)$. However, IPmax was higher in the study group in relation to the control group $(P=0.034)$. There was improvement in both groups in some variables, such as delay in the beginning of the pharyngeal phase and residues in valleculae, although without statistical significance. Conclusion: Inspiratory muscle training did not have significant effects on the biomechanics of swallowing.

Keywords: Bronchiectasis. Deglutition. Disorders Deglutition. Reabilitation. 


\section{INTRODUÇÃO}

A bronquiectasia é uma doença supurativa crônica, com alterações irreversíveis dos brônquios e persistente produção de escarro purulento e infecções respiratórias recorrentes (CHALMERS et al., 2018). Ocorre dilatação associada à inflamação, somado ao enfraquecimento da parede brônquica, que leva à distorção e à cicatrização nos brônquios, posto que, a longo prazo, traz prejuízos nos mecanismos de defesa e na depuração mucociliar com acúmulo de secreção no trato respiratório (ZENGLI, 2014). A doença apresenta como manifestações clínicas: pneumonia de repetição, tosse crônica, produção crônica e excessiva de secreção purulenta, dispneia, hemoptise, febre, fadiga e perda de peso (ZENGLI, 2014; CHALMERS et al., 2018).

Pela característica crônica e irreversível da bronquiectasia, a sua progressão pode influenciar outras funções, como a deglutição (OLIVEIRA, 2010; PICCIONE et al., 2012; GONÇALVES, 2015), tendo em vista que, para uma deglutição segura, realiza-se a apneia da respiração, a qual depende da integridade do sistema respiratório.

$\mathrm{Na}$ bronquiectasia ocorre perda da força muscular respiratória e diminuição da capacidade pulmonar (GUIMARÃES et al., 2012; PICCIONE et al., 2012). É provável que a pressão de ar subglótica encontre-se diminuída, alterando a proteção da via aérea inferior durante a deglutição, levando à disfagia (HAMMOND, 2008), fato que reforça a necessidade de avaliação fonoaudiológica adequada dessa função.

Poucos são os trabalhos encontrados na literatura que relacionam bronquiectasia e disfagia (OLIVEIRA, 2010; PICCIONE et al., 2012; GONÇALVES, 2015). Oliveira (2010), ao avaliar a deglutição de sujeitos com essa doença, encontrou alteração na elevação do complexo hiolaríngeo durante a avaliação clínica, indicando alteração na fase faríngea da deglutição.

Salienta-se que, nesta população, nem sempre as alterações da função de deglutição podem ser identificadas somente pela avaliação clínica, e, para meIhor caracterização e identificação de possíveis alterações, o profissional precisa lançar mão de avaliação instrumental, como a videofluoroscopia da deglutição (VFD), pois é possível observar penetração e/ou aspiração laríngea na ausência de sinais clínicos indicativos dessas alterações (OLIVEIRA, 2010).

A VFD é utilizada na identificação de possíveis alterações, por meio da observação detalhada das estruturas anatômicas e a relação temporal dos fenômenos ocorridos nas fases oral e faríngea durante a ingestão de alimentos com diferentes consistências e volumes (ANÉAS; DANTAS, 2014). Na população com bronquiectasia e demais doenças pulmonares, é importante basear-se nos achados das avaliações clínica e instrumental a fim de elaborar um planejamento terapêutico adequado que, muitas vezes, permeia uma atuação multidisciplinar.

Uma modalidade terapêutica na atuação com essa população, que vem despertando interesse na literatura científica, é o treinamento muscular respiratório, o qual objetiva, por meio de exercícios de condicionamento muscular com equipamentos direcionados, fortalecer a musculatura periférica e respiratória tanto expiratória quanto inspiratória (OZALP et al., 2012).

O Treinamento Muscular Inspiratório (TMI) é realizado quando os músculos inspiratórios estão fracos, uma vez que o esforço máximo pode não ser suficiente para expandir os pulmões (NASCIMENTO; MAIWORM; CADER, 2013). O TMI também pode diminuir o tempo inspiratório e aumentar o tempo de exalação pela melhora da resistência muscular (OZALP et al., 2019), com possível melhora na coaptação glótica e proteção da via aérea. Destaca-se, a partir disso, a necessária relação do trabalho muscular respiratório e a fisiologia da deglutição, na qual os mesmos músculos são envolvidos.

Baseado, então, nos poucos estudos com adultos na literatura, como de Oliveira (2010) e Gonçalves (2015), que identificaram alterações na biomecânica da deglutição, tanto na avaliação clínica quanto instrumental, na carência de trabalhos sobre o efeito do TMR na função de deglutição (PITTS et al., 2009) e nenhum trabalho sobre os efeitos do TMI nesta função, o presente trabalho teve por objetivo analisar os efeitos do TMI na deglutição de sujeitos com bronquiectasia.

\section{MÉTODO}

Estudo caracterizado por ensaio clínico randomizado, não cegado, realizado em Hospital Universitário no interior do Rio Grande do Sul, previamente aprovado pelo Comitê de Ética em Pesquisa da instituição conforme a resolução 466/2012, com parecer número 1.138.094. Os participantes do estudo receberam todos os esclarecimentos necessários sobre a pesquisa e, após, assinaram o Termo de Consentimento Livre e Esclarecido.

A população do estudo foi de sujeitos com diagnóstico de bronquiectasia que foram encaminhados ao ambulatório de Fisioterapia, setor de reabilitação pulmonar. Aos sujeitos foram explicados como seria o tratamento e os mesmos foram convidados a participar da pesquisa de forma voluntária. 
Para a participação no estudo os sujeitos não poderiam ter realizado tratamentos fonoaudiológicos, reabilitação pulmonar ou treinamento muscular inspiratório prévios, deveriam estar em estado clínico e sinais vitais estáveis, além de apresentar valores do miniexame do Estado Mental (MEEM) dentro do esperado, tendo como base o nível de escolaridade (BRUCKI et al., 2003). Além disso, os pacientes não poderiam relatar ou ter diagnóstico de alterações neurológicas, neoplasias, realização de algum tipo de cirurgia orofaríngea/laringotraqueal, realização de radioterapia e utilização de medicamentos que pudessem comprometer o nível de consciência e prejudicar a fidedignidade das avaliações.

A amostra foi composta por 14 sujeitos, posto que dois desistiram durante a realização das avaliações e um desistiu durante a aplicação dos protocolos de fisioterapia, finalizando a amostra com 11 sujeitos que foram randomizados em grupo controle (GC), o qual contou com cinco participantes, e grupo estudo (GE), composto por seis indivíduos. Ressalta-se que não foi possível atingir a amostra conforme o cálculo amostral havia estimado, pois durante a coleta diminuiu o número de pacientes encaminhados, houve dificuldade com espaço físico para a coleta e, ainda, teve pacientes que tiraram o consentimento de participação na pesquisa.

O diagnóstico de bronquiectasia foi confirmado por meio da história clínica e radiografia de tórax e confirmado por tomografia computadorizada, e os indivíduos realizaram teste de função pulmonar para identificar outras comorbidades.

No GC os pacientes realizaram fisioterapia convencional e no GE os pacientes realizaram fisioterapia convencional associada ao TMI; ambos os grupos realizaram o protocolo duas vezes por semana, durante quatro semanas (SAPIENZA; TROCHE; PITTS, 2011) no ambulatório de fisioterapia, sendo atendidos por profissionais fisioterapeutas ou por acadêmicos, sob supervisão do docente.

\section{Avaliação da biomecânica da deglutição}

A avaliação videofluoroscópica foi realizada no setor de radiologia do Hospital Universitário, executada pelo técnico em radiologia e pelo fonoaudiólogo (BARROS; SILVA; DE-ANGELIS, 2010). As consistências utilizadas no exame foram: Líquido: $15 \mathrm{ml}$ líquido + $15 \mathrm{ml}$ bário; Pudim/pastoso: $15 \mathrm{ml}$ líquido $+15 \mathrm{ml}$ bário +1 colher e meia de $3 \mathrm{ml}$ de espessante e o contraste

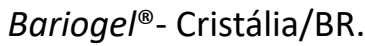

As imagens de videofluoroscopia foram obtidas com o equipamento marca Siemens, modelo Axiom Iconos R200, sendo capturadas e gravadas por meio do software ZScan6 Gastro - Versão: 6.1.2.11, instalado no computador Itautec Infoway, Windows 7, processador Intel Pentium P6200, sendo o registro da imagem realizado em 30 quadros/segundo e com qualidade ideal para o estudo das regiões visualizadas (COSTA, 2010).

O foco da imagem videofluoroscópica foi definido anteriormente pelos lábios, superiormente pelo palato duro, posteriormente pela parede posterior da faringe e inferiormente pela bifurcação da via aérea e esôfago, na altura da 7a vértebra cervical, sendo o exame realizado no período de tempo mais curto possível para diminuir a exposição do paciente à radiação (BARROS; SILVA DE-ANGELIS, 2010). O diagnóstico da deglutição foi realização pela classificação de Padovani et al. (2007).

A biomecânica da deglutição foi analisada por intermédio das seguintes variáveis:

- Variáveis visuoperceptuais (BAIJENS et al., 2011)

- Escape posterior precoce (pre-swallow posterior spill) (perda prematura do bolo para faringe): 0 - ausência; 1 - traço; 2 - > traço; 3 - > 50\% do bolo; 4 - todo bolo vai para a faringe antes da deglutição.

- Número de deglutições (piece-meal deglutition) (número de vezes que o bolo alimentar é fragmentado): 0 - ausência de deglutição; 1 - uma deglutição; 2 - duas deglutições; 3 - três deglutições; 4 - quatro ou mais deglutições.

- Atraso do início da fase faríngea da deglutição (delayed initiation pharyngeal reflex) (momento em que ocorre o disparo da fase faríngea): 0 - normal ou base de língua; 1 entre a base de língua e valécula; 2 - abaixo da valécula.

- Resíduo em valécula (post swallow vallecular pooling) (estase do bolo alimentar em valécula após a deglutição completa): 0 não houve estase ou mínima estase; 1 - mínima estase ou resíduo preenche até $50 \%$ da valécula; 2 - resíduo preenche mais de $50 \%$ da valécula.

- Resíduo em recessos piriformes (post swallow pyriform sinus pooling) (estase do bolo alimentar em recessos piriformes após 
a deglutição completa): 0 - não houve estase; 1 - leve a moderada estase; 2 - grave estase, preenchendo os recessos piriformes.

- Penetração/Aspiração: 0 - normal; 1 - penetração - bolo em contato com via aérea inferior acima das pregas vocais; 2 - aspiração - bolo em contato com via aérea inferior passando o nível das pregas vocais.

Variáveis temporais (KAHRILAS et al., 1997)

- Tempo de trânsito oral (oral transition time): momento do primeiro movimento do bolo na cavidade oral para a faringe até o fechamento da junção glossopalatal;

- Tempo de trânsito faríngeo (Pharyngeal transition time): momento de abertura da junção glossopalatal até o fechamento do esfíncter esofágico superior.

Para a análise do movimento do osso hioide foram adotados como pontos de referência anatômicos fixos para a angulação dos movimentos do osso hioide, o processo mastoide e o processo alveolar dos dentes incisivos anteriores, e como ponto móvel o corpo do hioide. Foram analisadas: posição de repouso, elevação máxima e deslocamento (repouso - elevação máxima) (BILHERI, 2016).

As variáveis da deglutição foram analisadas no software Kinovea ${ }^{\circledR}$ (versão 8.20, 2012). 0 Kinovea ${ }^{\circledR}$ é um software de acesso gratuito, traduzido para mais de 18 idiomas, incluindo o português, aplicável para análises do movimento humano e posturas, permitindo, além de outros recursos, a verificação de angulações durante o movimento (BILHERI, 2016).

A análise da avaliação videofluoroscópica foi realizada individualmente por três fonoaudiólogos juízes com experiência na área de disfagia. Os profissionais foram cegados em relação aos objetivos da pesquisa, à identificação dos sujeitos, bem como à avaliação dos demais juízes.

\section{Avaliação Fisioterapêutica}

A avaliação da força muscular respiratória foi realizada por meio da manovacuometria por fisioterapeuta, com equipamento de manovacuômetro digital modelo MVD300, graduado em $\mathrm{cmH}_{2} \mathrm{O}$. A manovacuometria foi feita com indivíduo sentado, e foram realizadas pelo menos cinco mensurações de cada pressão, com um minuto de intervalo entre cada repetição e tempo mínimo de sustentação de 1,5 segundo em cada manobra.
Nesta avaliação deveria haver pelo menos três medidas reprodutíveis (uma com variação igual ou inferior a $10 \%$ e a outra com variação de, no máximo, $20 \%$ com a pressão de maior valor). A pressão de maior valor, tanto da PImáx quanto PEmáx, foi adotada no estudo como força muscular respiratória do indivíduo (PESSOA et al., 2014).

\section{Fisioterapia convencional e treinamento muscular inspiratório}

A fisioterapia convencional e o treinamento muscular respiratório foram realizados por profissional fisioterapeuta com experiência na área.

Os participantes do GC realizaram tratamento fisioterapêutico convencional no Ambulatório de Fisioterapia duas vezes por semana com duração de 50 minutos cada sessão durante quatro semanas.

A fisioterapia convencional compreendeu as técnicas de desobstrução brônquica, compostas por: inaloterapia, Vibração-Vibrocompressão, tosse assistida e aumento do fluxo expiratório e drenagem autógena (BRANT; REIS; VIEIRA, 2009; FLUDE; AGENT; BILTON, 2012; NASCIMENTO; MAIWORM; CADER, 2013).

Os participantes do GE realizaram tratamento fisioterapêutico convencional associado ao TMI duas vezes por semana, com duração de 1 hora e 10 minutos cada sessão durante quatro semanas (TOUT; TAYARA; HALIMI, 2013).

O dispositivo utilizado para o TMI foi o de carga resistiva POWERbreathe ${ }^{\circledR}$ modelo medic plus. A carga estabelecida foi de $30 \%$ da manovacuometria, com duração de 21 minutos cada treino (DALL'AGO et al., 2006). Os sujeitos do GE fizeram a manovacuometria semanalmente, quando, então, foi ajustada a carga do incentivador de $30 \%$ do valor da manovacuometria.

\section{Análise dos dados}

Os dados foram armazenados em planilha Excel e analisados com o pacote estatístico SPSS 21.0. A normalidade da distribuição dos dados numéricos foi verificada por meio do teste de Kolmogorov Smirnov.

A descrição dos dados foi feita por meio de média e desvio padrão para as variáveis numéricas, e frequência absoluta e relativa para as variáveis categóricas. Equações de Estimação Generalizadas e o teste Post Hoc de Bonferroni foram utilizados para comparar as variáveis pré e pós-intervenção de cada grupo. Foram consideradas significativas as análises com $\mathrm{p}<0,05$. 
A concordância entre os avaliadores foi verificada por meio do Coeficiente de Correlação Intraclasse, classificado, segundo Fleiss e Cohen (1973), como fraco $(<0,4)$, regular $(0,4-0,75)$ e excelente $(0,75-1,00)$ para as variáveis numéricas, e do Coeficiente Kappa, classificado segundo Landis e Koch (1977), os quais propõem seis categorias: ruim (0), fraca $(0,01$ a 0,20$)$, regular $(0,21$ a 0,40$)$, moderada $(0,41$ a 0,60$)$, substancial $(0,61$ a 0,80$)$ e quase-perfeita $(>0,80)$ para as variáveis categóricas.

Já para o grau de repouso: elevação máxima e deslocamento do hioide pré na consistência líquida; grau de deslocamento do hioide pós na consistência líquida; tempo de trânsito faríngeo pré e pós na consistência pudim; no grau de repouso do hioide pós na consistência pudim a concordância foi regular; grau de elevação máxima do hioide pré e pós na consistência pudim; grau de deslocamento do hioide pós na consistência pudim concordância regular $(0,4-0,75)$. Consistência fraca foi observada entre as medidas de tempo de trânsito faríngeo pós na consistência líquida $(<0,4)$ (Tabela 1).

Para a consistência líquida, a concordância entre os avaliadores foi quase perfeita para as variáveis categóricas: número de deglutições pós, atraso do início da fase faríngea da deglutição pós, resíduo em valécula pré e pós, resíduos em recessos piriformes pré e pós e penetração/aspiração pré e pós $(>0,80)$. A concordância foi substancial para as variáveis: escape posterior precoce pré e pós, número de deglutições pré e atraso do início da fase faríngea da deglutição $(0,61-0,80)$.

Na consistência pudim, a concordância entre os avaliadores foi quase perfeita para as variáveis: escape posterior precoce, pré e pós, resíduo em valécula pré e pós, resíduos em recessos piriformes pós, e penetração/aspiração pré e pós $(>0,80)$; substancial para as variáveis: número de deglutições pré e pós, atraso do início da fase faríngea da deglutição pré e pós e resíduos em processos piriformes $(0,61-0,80)$ (Tabela 2).

No presente estudo a videofluoroscopia serviu de base para analisar, por meio de parâmetros quantitativos e qualitativos, os efeitos do treinamento muscular inspiratório na função de deglutição. Pode-se observar que houve variação entre as concordâncias das variáveis categóricas e numéricas. Alguns autores ressaltam que, dentre os fatores que determinam uma boa concordância, estão a instrução, o treinamento metodológico e a experiência dos avaliadores a respeito da biomecânica normal e alterada da deglutição, embora a subjetividade individual não possa ser excluída, principalmente nas variáveis avaliador-dependente (BAIJENS et al., 2011; GONÇALVES, 2015).

Tabela 1 - Confiabilidade e concordância entre medidas da videofluoroscopia obtidas por três avaliadores ( $N=11$ )

\begin{tabular}{|c|c|c|c|c|}
\hline \multirow[b]{2}{*}{ Medidas } & \multicolumn{4}{|c|}{ Nível de confiabilidade } \\
\hline & $\begin{array}{c}\text { Pré-intervenção } \\
\text { CCI (IC95\%) }\end{array}$ & $\mathbf{p}$ & $\begin{array}{c}\text { Pós-intervenção } \\
\text { CCI (IC95\%) }\end{array}$ & $\mathbf{P}$ \\
\hline \multicolumn{5}{|l|}{ Consistência líquida } \\
\hline Tempo de trânsito oral em segundos & $0,96(0,90-0,99)$ & $<0,001$ & $0,98(0,91-0,99)$ & $<0,001$ \\
\hline Tempo de trânsito faríngeo em segundos & $0,75(0,17-0,93)$ & $<0,001$ & $-0,03(-0,57-0,55$ & 0,515 \\
\hline Repouso do hioide $\left(^{\circ}\right)$ & $0,52(-0,29-0,86)$ & 0,076 & $0,76(0,38-0,93)$ & 0,002 \\
\hline Elevação máxima do hioide $\left({ }^{\circ}\right)$ & $0,74(0,34-0,92)$ & 0,002 & $0,76(0,34-0,93)$ & 0,004 \\
\hline Deslocamento do hioide $\left({ }^{\circ}\right)$ & $0,68(0,07-0,91)$ & 0,019 & $0,5890,02-0,87)$ & 0,010 \\
\hline \multicolumn{5}{|l|}{ Consistência pudim } \\
\hline Tempo de trânsito oral em segundos & $0,96(0,89-0,99)$ & $<0,001$ & $0,97(0,92-0,99)$ & $<0,001$ \\
\hline Tempo de trânsito faríngeo em segundos & $0,44(-0,14-0,81)$ & 0,046 & $0,54(-0,05-0,85)$ & 0,002 \\
\hline Repouso do hioide $\left(^{\circ}\right)$ & $0,82(0,45-0,95)$ & $<0,001$ & $0,69(0,20-0,91)$ & 0,002 \\
\hline Elevação máxima do hioide $\left({ }^{\circ}\right)$ & $0,72(0,29-0,92)$ & 0,003 & $0,66(-0,01-0,90)$ & 0,025 \\
\hline Deslocamento do hioide $\left({ }^{\circ}\right)$ & $0,88(0,65-0,96)$ & $<0,001$ & $0,61(0,06-0,88)$ & 0,014 \\
\hline
\end{tabular}


Tabela 2 - Proporção de concordâncias entre variáveis da videofluoroscopia obtidas por três avaliadores (N=11)

\begin{tabular}{lcc}
\hline Variáveis & \multicolumn{2}{c}{ Nível de concordância } \\
\cline { 2 - 3 } & $\begin{array}{c}\text { Pré-intervenção } \\
\text { CCI (IC95\%) }\end{array}$ & $\begin{array}{c}\text { Pós-intervenção } \\
\text { CCI (IC95\%) }\end{array}$ \\
\hline Consistência líquida & $0,77(0,54-1,00)$ & $0,77(0,54-1,00)$ \\
Escape posterior precoce & $0,77(0,54-1,00)$ & $0,85(0,65-1,00)$ \\
Número de deglutições & $0,77(0,54-1,00)$ & $0,85(0,65-1,00)$ \\
Atraso do início da fase faríngea da deglutição & $0,92(0,78-1,00)$ & $1,00(1,00-1,00)$ \\
Resíduo em valécula & $0,92(0,78-1,00)$ & $0,85(0,65-1,00)$ \\
Resíduo em recessos piriformes & $1,00(1,00-1,00)$ & $1,00(1,00-1,00)$ \\
Penetração/aspiração & & $0,85(0,65-1,00)$ \\
Consistência pudim & $0,92(0,78-1,00)$ & $0,77(0,54-1,00)$ \\
Escape posterior precoce & $0,77(0,54-1,00)$ & $0,66(0,37-0,95)$ \\
Número de deglutições & $0,62(0,36-0,88)$ & $0,92(0,78-1,00)$ \\
Atraso do início da fase faríngea da deglutição & $0,92(0,78-1,00)$ & $0,92(0,78-1,00)$ \\
Resíduo em valécula & $0,77(0,54-1,00)$ & $1,00(1,00-1,00)$ \\
Resíduo em recessos piriformes & $1,00(1,00-1,00)$ & \\
Penetração/aspiração & &
\end{tabular}

Coeficiente Kappa.

Nota: IC95\%: Intervalo de Confiança de 95\%.

\section{RESULTADOS}

Foram avaliados 11 indivíduos com bronquiectasia, a maioria com deglutição normal $(63,6 \%)$, adultos de meia idade $(54,5 \%)$ do sexo feminino $(72,7 \%)$, com média do MEEM de $24,5 \pm 2,3$ pontos (Tabela 3 ).
Tanto no grupo controle quanto no grupo estudo, a pressão inspiratória máxima foi maior após a intervenção $(p=0,007)$.

Tabela 3 - Características prévias da amostra de sujeitos com bronquiectasia (N=11)

\begin{tabular}{lc}
\hline Características & N $(\mathbf{\%})$ \\
\hline Sexo & \\
Feminino & $8(72,7)$ \\
Masculino & $3(27,3)$ \\
Faixa etária em anos & $3(27,3)$ \\
19 a 44 & $6(54,5)$ \\
45 a 64 & $2(18,2)$ \\
$\geq 65$ & $24,5 \pm 2,3$ \\
MEEM (média \pm DP pontos) & \\
Diagnóstico clínico da deglutição & $7(63,6)$ \\
Normal & $4(36,4)$ \\
Funcional & \\
\hline
\end{tabular}

MEEM: Mini Exame do Estado Mental 
Tabela 4 - Comparação da avaliação respiratória pré e pós-intervenção, entre os grupos (N=11)

\begin{tabular}{lccccc}
\hline & \multirow{2}{*}{$\begin{array}{c}\text { Grupo Controle } \\
(\mathbf{N}=\mathbf{5})\end{array}$} & $\begin{array}{c}\text { Grupo } \\
\text { Intervenção } \\
(\mathbf{N}=\mathbf{6})\end{array}$ & $\mathbf{G r u p o}$ & Tempo & $\begin{array}{c}\text { Grupo vs. } \\
\text { Tempo }\end{array}$ \\
\cline { 5 - 6 } & & & & & \\
\hline Pressão inspiratória máxima $\left(\mathrm{cmH}_{2} \mathrm{O}\right)$ & $61,6^{\mathrm{A}} \pm 7,6$ & $77,5^{\mathrm{A}} \pm 13,2$ & $\mathbf{0 , 0 3 4}$ & $\mathbf{0 , 0 0 7}$ & 0,058 \\
T0 & $66,0^{\mathrm{Ba}} \pm 8,1$ & $115,5^{\mathrm{Bb}} \pm 18,3$ & & & \\
T1 & & & & & \\
Pressão expiratória máxima $\left(\mathrm{cmH}_{2} \mathrm{O}\right)$ & $70,0^{\mathrm{a}} \pm 5,0$ & $98,3^{\mathrm{b}} \pm 9,0$ & $\mathbf{0 , 0 0 2}$ & 0,246 & 0,192 \\
T0 & $69,4^{\mathrm{a}} \pm 107$ & $113,8^{\mathrm{b}} \pm 9,9$ & & & \\
T1 &
\end{tabular}

Equações de Estimação Generalizadas e Teste de Post Hoc de Bonferroni e Diferença Mínima Significativa.

Após a intervenção, a pressão inspiratória máxima foi superior no grupo estudo em relação ao grupo controle $(p=0,034)$. O comportamento entre os dois grupos foi similar ao longo do tempo (interação grupo vs. tempo).
Em relação à carga de treinamento, houve mudança estatística significativa na quarta semana entre o grupo controle e o grupo estudo $(p<0,001)$. As demais medidas não apresentaram diferença em relação ao tempo e à interação grupo vs. tempo (Tabela 4).

Tabela 5 - Comparação das variáveis categóricas pré e pós-intervenção, entre os grupos ( $N=11)$

\begin{tabular}{|c|c|c|c|c|c|}
\hline \multirow[b]{2}{*}{ Variáveis } & \multirow{2}{*}{$\begin{array}{l}\text { Grupo Controle } \\
\qquad(\mathbf{N}=\mathbf{5})\end{array}$} & \multirow{2}{*}{$\begin{array}{c}\text { Grupo } \\
\text { Intervenção } \\
(\mathbf{N}=6)\end{array}$} & \multicolumn{3}{|c|}{$\mathbf{P}$} \\
\hline & & & Grupo & Tempo & $\begin{array}{c}\text { Grupo vs. } \\
\text { Tempo }\end{array}$ \\
\hline \multicolumn{6}{|l|}{ Diagnóstico T0 } \\
\hline Deglutição normal & $3(60,0)$ & $4(66,7)$ & \multirow{5}{*}{$<0,001$} & \multirow{5}{*}{$\S$} & \multirow{5}{*}{$\S$} \\
\hline Deglutição funcional & $2(40,0)$ & $2(33,3)$ & & & \\
\hline Diagnóstico T1 & & & & & \\
\hline Deglutição normal & $3(60,0)$ & $6(100,0)$ & & & \\
\hline Deglutição funcional & $2(40,0)$ & $0(0,0)$ & & & \\
\hline \multicolumn{6}{|l|}{ FOIS T0 } \\
\hline VO total sem restrições & $5(100,0)$ & $6(100,00)$ & \multirow{3}{*}{$\S$} & \multirow{3}{*}{$\S$} & \multirow{3}{*}{$\S$} \\
\hline FOIS T1 & & & & & \\
\hline VO total sem restrições & $5(100,0)$ & $6(100,00)$ & & & \\
\hline \multicolumn{6}{|l|}{ Videofluoroscopia T0 } \\
\hline Deglutição normal & $1(20,0)$ & $2(33,3)$ & \multirow{6}{*}{0,179} & \multirow{6}{*}{$\mathbf{0 , 0 0 7}$} & \multirow{6}{*}{0,211} \\
\hline Deglutição funcional & $2(40,0)$ & $3(50,0)$ & & & \\
\hline Disfagia leve & $2(40,0)$ & $1(16,7)$ & & & \\
\hline \multicolumn{3}{|l|}{ Videofluoroscopia T1 } & & & \\
\hline Deglutição normal & $1(20,0)$ & $5(83,3)$ & & & \\
\hline Deglutição funcional & $4(80,0)$ & $1(16,7)$ & & & \\
\hline \multicolumn{6}{|l|}{ Rosenbek T0 } \\
\hline Contraste não entra na via aérea & $4(80,0)$ & $5(83,3)$ & \multirow{4}{*}{$\S$} & \multirow{4}{*}{0,999} & \multirow{4}{*}{$\S$} \\
\hline $\begin{array}{l}\text { Contraste até acima pregas vocais, sem } \\
\text { resíduo }\end{array}$ & $1(20,0)$ & $1(16,7)$ & & & \\
\hline \multicolumn{3}{|l|}{ Rosenbek T1 } & & & \\
\hline Contraste não entra na via aérea & $5(100,0)$ & $6(100,0)$ & & & \\
\hline
\end{tabular}

Equações de Estimação Generalizadas. § Dados não permitem o cálculo.

FOIS: Functional Oral Intake Scale. 
O diagnóstico da deglutição apresentou diferença entre os grupos. Após intervenção, $100 \%$ dos pacientes do grupo intervenção apresentaram deglutição normal, enquanto os do grupo controle mantiveram-se em $60 \%(p<0,001)$. No grupo intervenção, o resultado da videofluoroscopia apresentou diferença pré e pós intervenção. A frequência de indivíduos com deglutição normal passou de $33,3 \%$ para $83,3 \%$ $(P=0,007)$ (Tabela 5).

\section{DISCUSSÃO}

Participaram desta pesquisa 11 indivíduos com diagnóstico de bronquiectasia, adultos de meia idade $(54,5 \%)$ do sexo feminino $(72,7 \%)$ (Tabela 3). A literatura destaca que essa doença é mais comum no sexo feminino (ZENGLI, 2014; OLIVEIRA, 2010; QUINT et al., 2016). Quanto à faixa etária, os achados deste estudo vão ao encontro da literatura, uma vez que a bronquiectasia acomete mais sujeitos adultos (OLIVEIRA, 2010; QUINT et al., 2016).

A bronquiectasia apresenta, em sua fisiopatologia, enfraquecimento dos brônquios em razão da inflamação e infecção crônica e recorrente, associada à tosse produtiva e obstrução do fluxo aéreo (FLUDE; AGENT; BILTON, 2012; ZENGLI, 2014). A ocorrência desse processo modifica a mecânica ventilatória, pela perda da força muscular respiratória e alterações de volumes e capacidades pulmonares (GUIMARÃES et al., 2012; ZENGLI, 2014).

Dentre as possibilidades terapêuticas para a bronquiectasia tem-se a realização do treinamento muscular respiratório, que pode ser para musculatura expiratória ou inspiratória. O Treinamento Muscular Inspiratório (TMI), que foi realizado no presente estudo, visa a diminuir a fraqueza muscular inspiratória, a qual pode levar ao músculo discordância de carga e capacidade, e, assim, aumentar os sintomas de dispneia, intolerância ao exercício, hipoventilação e parada respiratória (OZALP et al., 2019).

A pesquisa de Newall, Stockley e Hill (2005), verificou os efeitos do TMI em sujeitos com bronquiectasia em um programa de oito semanas com grupo controle e estudo. Os autores verificaram aumento estatisticamente significativo na PImax após o TMI nos sujeitos do grupo estudo. $O$ mesmo foi encontrado no trabalho de Liaw et al. (2011), entretanto não houve efeito sobre a função respiratória e na qualidade de vida.

A pesquisa de Hoffman et al. (2018) realizou TMI em pacientes com doença pulmonar crônica, incluindo bronquiectasia. $\mathrm{O}$ treinamento de alta intensi- dade, utilizando o POWERbreathe ${ }^{\circledR}$, foi realizado, diariamente, em casa. Os autores ressaltaram que o TMI pode melhorar a capacidade de trabalho muscular com consequente melhoria da resposta ventilatória. Tais resultados também foram encontrados no trabaIho de Ozalp et al. (2019).

Nesta pesquisa, o grupo controle realizou apenas terapia convencional, enquanto o grupo estudo trabalhou a terapia tradicional associada ao TMI durante quatro semanas. Pode-se verificar que em ambos os grupos a PImáx foi maior após intervenção $(p=0,007)$. A PImáx, entretanto, foi superior no grupo estudo em relação ao grupo controle $(p=0,034)$. O comportamento entre os dois grupos foi similar ao longo do tempo (interação grupo vs. tempo) (Tabela 4).

Assim, com os resultados encontrados nesta pesquisa e em outros estudos com metodologias semelhantes, é possível inferir que os sujeitos com bronquiectasia são beneficiados do ponto de vista do aumento da força muscular inspiratória quando realizaram o TMI. Não foram encontrados, entretanto, outros estudos que tivessem o objetivo de verificar os efeitos do TMI sobre a deglutição nesta população.

Machado et al. (2015) analisou o efeito do TME e TMI com o Respiron ${ }^{\circledR}$ sobre a função de deglutição em sujeitos normais por meio da videofluoroscopia. Os pesquisadores encontraram como resultado do TME e TMI diminuição do tempo de trânsito faríngeo.

Estudos demonstram que funções extrapulmonares, como a função de deglutição, podem estar comprometidas na bronquiectasia (OLIVEIRA, 2010; PICCIONE et al., 2012; GONÇALVES, 2015). Na função deglutitória da laringe, é necessário um adequado fluxo aéreo vindo dos pulmões para gerar a pressão subglótica suficiente que cause o fechamento máximo de forma esfinctérica da laringe para que haja adequada proteção da via aérea inferior (GONÇALVES, 2015).

Na comparação das frequências das medidas categóricas de avaliação videofluoroscópica, não foram observadas alterações estatisticamente significativas entre os grupos, os tempos e grupo vs. tempo (interação). Tal achado sugere que para essa amostra o TMI não foi efetivo para gerar mudanças estatisticamente significativas na biomecânica da deglutição, e não foi encontrado nenhum estudo com objetivo semelhante para comparar os resultados deste trabalho.

Cabe ressaltar que algumas variáveis, como atraso do início da fase faríngea e resíduos em valéculas, melhoraram nas duas consistências analisadas em ambos os grupos. 
No que se refere à variável atraso do início da fase faríngea (Delay initial pharyngeal reflex), foi evidenciada anteriorização do disparo da deglutição, de modo que, antes da terapia, o mesmo iniciava entre a base de língua e valéculas, passando a ocorrer na posição normal ou em base de língua. Em trabalho anterior (GONÇALVES, 2015) também encontrou início da fase faríngea entre base de língua e valéculas.

De acordo com Daniels e Foundas (2001), o local de início da fase faríngea da deglutição está relacionado ao aumento do risco de aspiração. Assim, o risco é menor quando o disparo ocorre na altura do ramo da mandíbula, risco moderado quando ocorre entre o ramo da mandíbula e valécula e maior quando o disparo ocorre abaixo da valécula.

$\mathrm{Na}$ variável presença de estase em valéculas, também foi evidenciada diminuição dos resíduos em ambos os grupos. A literatura traz que a presença de estase pode resultar em aspiração após a deglutição, pois o acúmulo de resíduo na faringe pode ser aspirado quando a respiração é retomada após a deglutição (MATSUO; PALMER, 2008).

Após a aplicação dos programas de reabilitação pulmonar, $100 \%$ dos pacientes do grupo estudo apresentaram deglutição normal, enquanto os do grupo controle mantiveram-se em $60 \%(p<0,001)$ (Tabela 5).

No grupo estudo, ainda, o resultado da videofluoroscopia apresentou diferença pré e pós-intervenção. A frequência de indivíduos com deglutição normal passou de 33,3\% para 83,3\% ( $p=0,007$ ) (Tabela 5). Esses achados podem estar relacionados às pequenas mudanças encontradas na biomecânica da deglutição, como diminuição das estases e mudança no local de disparo da deglutição, que, mesmo não sendo significantes, possibilitaram mudança na classificação da deglutição.

\section{CONCLUSÃO}

Neste trabalho a realização do treinamento muscular inspiratório não gerou efeitos sobre a biomecânica da deglutição, uma vez que modificações sutis foram evidenciadas em ambos os grupos testados. Encontrou-se aumento das pressões máximas após a intervenção, ratificando os benefícios já documentados do treinamento muscular nesta população.

Dentre as limitações deste estudo estão a variabilidade da idade dos sujeitos que compuseram a amostra, além de que a utilização de outras escalas, para avaliação da biomecânica da deglutição, poderia conferir maior sensibilidade à identificação de alterações dessa função, trazendo maior especificidade aos resultados.

Sugere-se a realização de pesquisas semelhantes com amostras maiores e uso de escalas com medidas padronizadas mais completas.

\section{REFERÊNCIAS}

ANÉAS, G. C. G.; DANTAS, R. O. A videofluoroscopia da deglutição na investigação da disfagia oral e faringeana. J Port Gastroenterol., v. 21, n. 4, p. 21-25, 2014.

BAIJENS, L. W. et al. Swallowing in Parkinson Patients versus Healthy Controls: Reliability of Measurements in Videofluoroscopy. Gastroenterol Res Pract., v. 2.011, n. 380.682, p. 1-9, 2011.

BARROS, A. P. B.; SILVA, S. A. C.; DE-ANGELIS, E. C. Videofluoroscopia da deglutição orofaríngea. In: JOTZ, G. P.; DE-ANGELIS, E. C.; BARROS, A. P. B. Tratado de deglutição e disfagia: no adulto e na criança. Rio de Janeiro: Revinter, 2010. p. 84-8. Cap. 11.

BILHERI, D. F. D. Medidas de excursão laríngea pré e pós exercícios respiratórios em indivíduos normais. 2016. Dissertação (Mestrado em Distúrbios da Comunicação Humana) - Universidade Federal de Santa Maria, Departamento de Fonoaudiologia, Santa Maria, 2016.

BRANT, T. C. S.; REIS, A. F. C.; VIEIRA, C. O. L. Técnicas modernas de desobstrução brônquica utilizadas em adultos. São Paulo: Manole, 2009.

BRUCKI, S. M. D. et al. Suggestions for utilization of the mini-mental state examination in Brazil. Arq Neuropsiquiatr., v. 61, n. 3-B, p. 777-781, 2003.

COSTA, M. M. B. Videofluoroscopia: método radiológico indispensável para a prática médica. Radiol Bras., v. 43, n. 2, p. VII-VIII, 2010.

CHALMERS, J. D. et al. Bronchiectasis. Nat Rev. Dis Primers., v. 4, n. 1, p. 1-18, 2018.

DALL'AGO, P. et al. Inspiratory muscle training in patients with heart failure and inspiratory muscle weakness: a randomized trial. J Am Coll Cardiol., v. 47, n. 4, p. 757-763, 2006.

DANIELS, S. K.; FOUNDAS, A. L. Swallowing physiology of sequential straw drinking. Dysphagia, v. 16, n. 3, p. 176-182, 2001.

FLEISS, J. L.; COHEN, J. The equivalence of weighted Kappa and the intraclass correlation coeficient as a measures of reliability. Education Psychol Measurem., v. 33, n. 3, p. 613619, 1973.

FLUDE, L. J.; AGENT, P.; BILTON, D. Chest Physiotherapy Techniques in Bronchiectasis. Clin Chest Med., v. 33, n. 2, p. 351-361, 2012.

GONÇALVES, B. F. T. Análise da biomecânica da deglutição em portadores de bronquiectasia. 2015. Dissertação (Mestrado em Distúrbios da Comunicação Humana) - Universidade Federal de Santa Maria, Departamento de Fonoaudiologia, Santa Maria, 2015. 
GUIMARÃES, F. S. et al. Efeitos da ELTGOL e do Flutter ${ }^{\circledR}$ nos volumes pulmonares dinâmicos e estáticos e na remoção de secreção de pacientes com bronquiectasia. Rev. Bras. Fisioter., v. 16, n. 2, p. 108-113, 2012.

HAMMOND, C. S. Cough and aspiration of food and liquids due to oral pharyngeal dysphagia. Lung., v. 186, n. Suppl 1, p. S35-40, 2008.

HOFFMAN, M. et al. The effects of inspiratory muscle training based on the perceptions of patients with advanced lung disease: a qualitative study. Braz J Phys Ther., v. 22. n. 3, p. 215-221, 2018.

KAHRILAS, P. J. et al. Impaired deglutitive airway protection: a videofluoroscopic analysis of severity and mechanism. Gastroenterol., v. 113, n. 5, p. 1.457-1.464, 1997.

LANDIS, J. R.; KOCH, G. G. The measurement of observer agreement for categorical data. Biometrics. v. 33, n. 1, p. 159-174, 1977.

LIAW, M. Y. et al. Inspiratory muscle training in bronchiectasis patients: a prospective randomized controlled study. Clin Rehabil., v. 25, n. 6, p. 524-536, 2011.

MACHADO, J. R. S. et al. Efeitos do exercício muscular respiratório na biomecânica da deglutição de indivíduos normais. Rev. Cefac., v. 17, n. 6, p. 1.909-1.915, 2015.

MATSUO, K.; PALMER, J. B. Anatomy and physiology of feeding and swallowing: normal and abnormal. Phys Med Rehabil Clin N Am., v. 19, n. 4, p. 691-707, 2008.

NASCIMENTO, B. S.; MAIWORM, A.; CADER, S. Força muscular respiratória e pico de fluxo expiratório de pacientes com bronquiectasia submetidos à reabilitação respiratória. Rev. Andal Med Deporte, v. 6, n. 2, p. 73-77, 2013.

NEWALL, C.; STOCKLEY, R. A.; HILL, S. L. Exercise training and inspiratory muscle training in patients with bronchiectasis. Thorax, v. 60, n. 11, p. 943-948, 2005.

OLIVEIRA, F. P. Análise do processo sinérgico da deglutição em pacientes portadores de bronquiectasia atendidos no Hospital Universitário Pedro Ernesto. 2010. Dissertação (Mestrado em Ciências Médicas) - Universidade do Estado do Rio de Janeiro, Rio de Janeiro, 2010.

OZALP, O. et al. High-intensity inspiratory muscle training in bronchiectasis: A randomized controlled trial. Respirology., v. 24, n. 3, p. 246-253, 2019.

OZALP, O. et al. Extrapulmonary features of bronchiectasis: muscle function, exercise capacity, fatigue, and health status. Multidiscip Respir Med., v. 7, n. 1, p. 1-6, 2012.

PADOVANI, A. R. et al. Protocolo fonoaudiológico de avaliação do risco para disfagia (PARD). Rev. Soc. Bras. Fonoaudiol., v. 12, n. 3, p. 199-205, 2007.

PESSOA, I. M. B. S. et al. Predictive equations for respiratory muscle strength according to international and Brazilian guidelines. Braz J Phys Ther., v. 18, n. 5, p. 410-418, 2014.

PITTS, T. et al. Impact of expiratory muscle strength training on voluntary cough and swallow function in parkinson disease. Chest., v. 135, n. 5, p. 1.301-1.308, 2009.

PICCIONE, J. C. et al. Bronchiectasis in Chronic Pulmonary Aspiration: Risk Factors and Clinical Implications. Pediatric Pulmonology, v. 47, n. 5, p. 447-452, 2012.
QUINT, J. K. et al. Changes in the incidence, prevalence and mortality of bronchiectasis in the UK from 2004 to 2013: a population-based cohort study. Eur Respir J., v. 47, n. 1, p. 186-193, 2016.

SAPIENZA, C.; TROCHE, M.; PITTS, T. Respiratory Strength Training: Concept and Intervention Outcomes. Seminars in Speech and Language, v. 32, n. 1, p. 21-30, 2011.

TOUT, R.; TAYARA, I.; HALIMI, M. The effects of respiratory muscle training on improvement of the internal and external thoraco-pulmonary respiratory mechanism in COPD patients. Annals of Physical and Rehabilitation Medicine, v. 56, n. 3, p. 193-211, 2013.

ZENGLI, W. Bronchiectasis: still a problem. Chin Med J., v. 127, n. 1, p. 157-172, 2014. 